\title{
CERÂMICA VERMELHA INCORPORADA COM PARTÍCULAS DE SÍNTER DE ACIARIA*
}

\section{Resumo}

\author{
Mônica Manhães Ribeiro ${ }^{1}$ \\ Eduardo Sousa Lima \\ André Ben-Hur da Silva Figueiredo ${ }^{3}$ \\ Verônica Scarpini Candido ${ }^{4}$ \\ Sergio Neves Monteiro \\ Carlos Maurício Fontes Vieira ${ }^{6}$ \\ Fernanda Santos da Luz
}

As partículas de sínter (PSs), retidas no precipitador eletrostático dos estágios de sinterização em uma planta de aciaria, foram incorporadas até $20 \%$ em peso na argila caulinítica para produzir cerâmicas vermelhas porosas melhoradas utilizadas na construção civil. As amostras foram preparadas por prensagem uniaxial e depois sinterizadas a 750,900 e $1050^{\circ} \mathrm{C}$, que são temperaturas típicas para produção de tijolos, blocos e telhas. Após o processamento, foram avaliadas as propriedades mecânicas e microestruturas relacionadas à porosidade dessas cerâmicas. Os resultados revelaram, pela primeira vez, como a porosidade pode ser associada à melhoria da resistência da cerâmica vermelha processada a temperaturas típicas para produção de tijolos, blocos e telhas. Observou-se também a melhoria dos isolamentos acústico e térmico devido à porosidade dessa cerâmica.

Palavras-chave: Partículas de sínter; Cerâmica vermelha porosa; Propriedades mecânicas.

\section{IMPROVED CLAY CERAMICS INCORPORATED WITH STEELMAKING SINTER PARTICLES}

\section{Abstract}

Sinter particles (SPs) retained in the electrostatic precipitator of the sintering stages in a steelmaking plant were incorporated up to $20 \mathrm{wt} \%$ into kaolinitic clay to produce improved porous ceramics for building construction. Specimens were prepared by uniaxial pressmolding and then fired at 750,900 and $1050^{\circ} \mathrm{C}$, which are typical temperatures for bricks, blocks and tiles production. Ceramic properties and microstructure related to porosity and mechanical strength were evaluated. The results disclosed, for the first time, how porosity might be associated with improved strength in clay ceramics processed at typical temperatures for bricks, blocks and tiles. Owing to the porosity, the ceramic thermal an acoustic insulation were also improved.

Keywords: Sinter particulates; Porous clay ceramic; Mechanical properties.

1 Graduada em Licenciatura em Ciências e matemática, M.C, Doutoranda em Ciência dos Materiais, Universidade Estadual do Norte Fluminense Darcy Ribeiro, UENF, Brasil.

2 Engenheiro Metalurgista D.C., Professor Titular, Seção de Engenharia Mecânica e de Materiais, Instituto Militar de Engenharia, Rio de Janeiro, RJ, Brasil.

3 Bacharel em Física, D.C., Professor Titular, Seção de Engenharia Mecânica e de Materiais, Instituto Militar de Engenharia, Rio de Janeiro, RJ, Brasil.

4 Bacharel em Ciências Biológicas, D.C, Professora adjunta, Faculdade de Engenharia de Materiais da Universidade Federal do Pará (UFPA), Ananindeua, PA, Brasil.

scarpini@ufpa.br

5 Engenheiro Metalurgista, Ph.D, Professor Titular, Seção de Engenharia Mecânica e de Materiais, Instituto Militar de Engenharia, Rio de Janeiro, RJ, Brasil.

6 Engenheiro Mecânico, D.C, Professor Associado, Engenharia Metalúrgica e de Materiais, Universidade Estadual do Norte Fluminense Darcy Ribeiro, UENF, Brasil. 
7 Engenheira de Materiais, M.C, Doutoranda em Ciência dos Materiais, Seção de Engenharia Mecânica e de Materiais, Instituto Militar de Engenharia, Rio de Janeiro, RJ, Brasil. fsl.santos@gmail.com 


\section{INTRODUÇÃO}

O estágio de sinterização de uma moderna planta de aciaria integrada é responsável pela produção do sínter utilizado como matéria-prima na fabricação de ferro gusa e aço [1]. Durante a sinterização de alta temperatura, uma nuvem de partículas é gerada e deve ser mantida para evitar a poluição atmosférica. Um precipitador eletrostático coleta essas partículas de sínter (PSs) por meio de potencial elétrico aplicado entre placas por onde essa nuvem é forçada a atravessar [2].

Em princípio, estas PSs poderiam ser recicladas dentro da mesma operação de sinterização. Entretanto, isto é tecnicamente inconveniente devido a possibilidade de recirculação de componentes alcalinos que são prejudiciais para o processo e podem resultar em uma queda na produtividade. A existência de substâncias alcalinas, concentradas nas PSs, atacarão os blocos refratários de alumina e então diminuir a vida operacional do revestimento do forno de sinterização [3].

Os resíduos industriais com uma quantidade relativamente alta de óxidos de ferro, como PSs coletadas no estágio de sinterização de uma indústria siderúrgica, estão sendo investigados para serem usados em cimento [4] e solos agrícolas [5]. Outra possibilidade de aplicação de resíduos contendo óxido de ferro é a sua incorporação em cerâmica vermelha para produtos de construção civil tais como, tijolos, blocos estruturais e telhas [6-9], as quais toleraram a incorporação de materiais com composições distintas. Em alguns casos, a incorporação pode contribuir para melhorar as propriedades cerâmicas [6-9]. Além disso, pode proporcionar uma economia para economia na quantidade de argila, que é um recurso natural não renovável [7]. Além disso, dependendo do tamanho de partícula do material incorporado, após a queima, a cerâmica vermelha adquire uma microestrutura porosa, o que pode aumentar o seu isolamento térmico e acústico.

Nesse contexto, o objetivo do presente trabalho foi caracterizar as partículas de sínter coletadas no estágio de sinterização de uma planta siderúrgica brasileira e avaliar o efeito da incorporação dessas partículas na cerâmica vermelha com propriedades avançadas.

\section{MATERIAIS E MÉTODOS}

Os materiais usados no presente trabalho foram: as partículas de sínter coletadas no estágio de sinterização da planta de aciaria ArcelorMittal Tubarão, aqui denotada como partículas de sinterização, PS, e um bloco de argila caolíntico, BC, usado para fabricar tijolos em uma indústria cerâmica localizada na cidade de Campos Dos Goytacazes, estado do Rio de Janeiro, Brasil. As características deste BC são dadas em outro lugar [10]. Resumidamente, é composta por caulinita, mica muscovita, quartzo e gibbsita, apresentando alta quantidade de $\mathrm{Al}_{2} \mathrm{O}_{2}$ e baixos fluxos, típicos das argilas a base de caulinita.

As PSs foram inicialmente caracterizadas em termos de composição química, física e mineralógica. A identificação qualitativa da fase mineralógica foi realizada em amostra em pó por difração de raios-X (DRX) usando um difratômetro Bruker-D4 Endeavor, operando com radiação Co-Ka (35 kV/40 mA) e um ângulo de varredura $2 \theta$ de 5 a $80^{\circ}$. A composição química foi determinada por fluorescência de raios- $X$ (FRX) em um equipamento Philips PW 2400. Para a determinação da distribuição do tamanho de partícula foram realizados métodos de peneiramento e sedimentação, seguindo a norma ABNT-NBR 7181/84 [11]. 
Foram realizadas incorporações de até $20 \%$ em peso de PS no bloco argiloso em um moinho de panela durante 30 minutos. As amostras retangulares de 114,5 x 2,54 x $10 \mathrm{~mm}$ foram obtidas por prensagem uniaxial a $18 \mathrm{MPa}$. Essas amostras foram secas a $110^{\circ} \mathrm{C}$ durante 24 horas e depois sinterizadas a 750,900 e $1050^{\circ} \mathrm{C}$ em um forno de laboratório, temperaturas essas tipicamente usadas no Brasil para a fabricação de tijolos de construção, blocos estruturais e telhas. A taxa de aquecimento foi de $3^{\circ} \mathrm{C} / \mathrm{min}$ com uma hora de imersão à temperatura máxima. $\mathrm{O}$ resfriamento ocorreu por convecção natural dentro do forno após seu desligamento. Essas amostras foram testadas quanto à quantidade de porosidade, bem como a sua resistência à flexão através do ensaio de flexão de três pontos. A porcentagem de porosidade foi determinada pela picnometria de $\mathrm{Hg}$, de acordo com um procedimento da norma ASTM C373-72 [12]. A força de ruptura de flexão de três pontos foi determinada em uma máquina de ensaio universal Instron 5582, usando uma velocidade de $0,5 \mathrm{~mm} / \mathrm{min}$. As análises de microscopia eletrônica de varredura (MEV) foram realizadas em um modelo Shimadzu SSX-550.

\section{RESULTADOS E DISCUSSÃO}

A Figura 1 mostra o padrão de DRX para a PS, que apresenta uma gama de composição mineralógica complexa e ampla devido às diferentes matérias-primas envolvidas no estágio de sinterização de uma planta de aciaria [3]. As principais fases cristalinas identificadas nesta figura foram: hematita $\left(\mathrm{Fe}_{2} \mathrm{O}_{3}\right)$, magnetita $\left(\mathrm{Fe}_{3} \mathrm{O}_{4}\right)$, calcita $\left(\mathrm{CaCO}_{3}\right)$, quartzo $\left(\mathrm{SiO}_{2}\right)$, dolomita $\left(\mathrm{Mg} . \mathrm{Ca} .\left(\mathrm{CO}_{3}\right)_{2}\right)$ e silvita $(\mathrm{KCl})$.

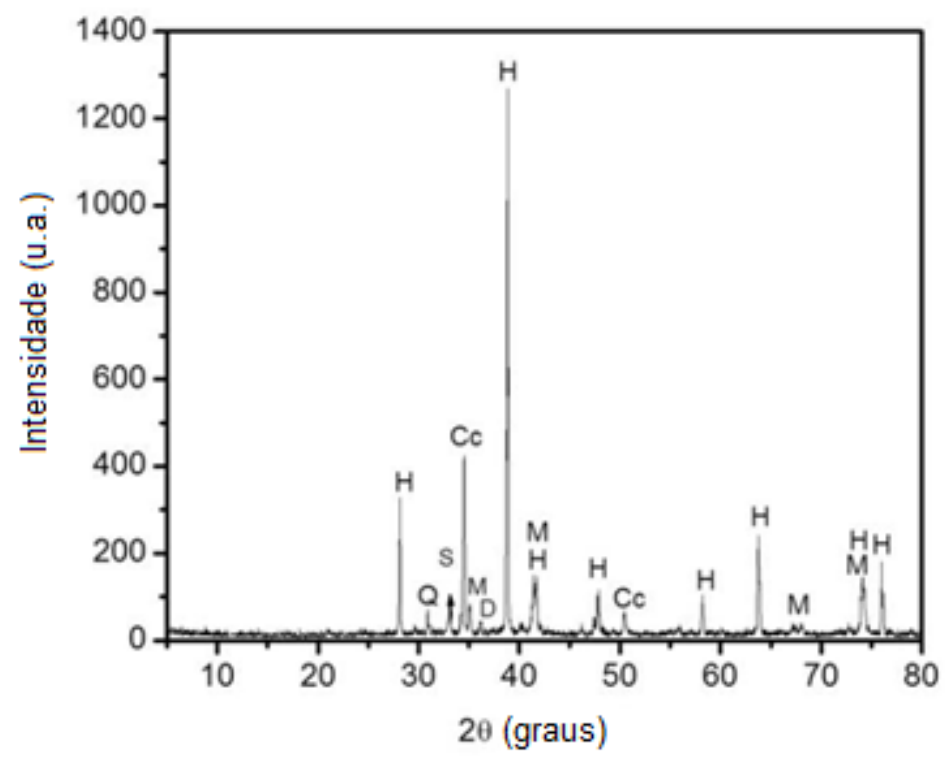

Figura 1. Padrão de DRX das partículas de sínter (PSs): $C c=$ calcita; $D=$ dolomita; $H=$ hematita; $M=$ magnetita; $\mathrm{Q}=$ quartzo; e $\mathrm{S}$ = silvita.

A Tabela 1 mostra a composição química da PS, que é predominantemente formada por compostos de ferro tais como, hematita e magnetita, também detectados pelo DRX (Fig. 1). Foi observada a ocorrência das fases $\mathrm{CaO}$ e $\mathrm{SiO}_{2}$ em quantidades relativamente elevadas. A perda ao fogo (PF) está predominantemente associada à combustão dos finos de coque, $\mathrm{C}$, que não foram identificados por difração de raios$\mathrm{X}$, bem como com a decomposição de calcita e dolomita. A pequena quantidade de 
$2,28 \%$ de $\mathrm{SO}_{3}$ sugere a presença de sulfatos, possivelmente de Ca, como o gesso, que também não foi identificado por difração de raios- $X$.

\begin{tabular}{|c|c|c|c|c|c|c|c|c|c|c|}
\hline Fe Total & $\mathrm{SiO}_{2}$ & $\mathrm{MnO}$ & $\mathrm{SO}_{3}$ & $\mathrm{Al}_{2} \mathrm{O}_{3}$ & $\mathrm{~K}_{2} \mathrm{O}$ & $\mathrm{MgO}$ & $\mathrm{CaO}$ & $\mathrm{ZnO}$ & $\mathrm{C}$ & $\mathrm{PF}^{*}$ \\
\hline 70.70 & 6.82 & 0.82 & 2.28 & 1.20 & 1.81 & 10.33 & 9.70 & 0.72 & 3.3 & 10.70 \\
\hline
\end{tabular}

Tabela 1. Composição química da PS (\% em peso).

A Figura 2 mostra a distribuição de tamanhos das PSs e sua relevância para a produção de diferentes materiais cerâmicos. De acordo com a Fig. 2a, a PS tem aproximadamente $80 \%$ do seu tamanho de partícula concentrado na faixa de 20 a $0,300 \mu \mathrm{m}$, o que é adequado para aplicação em cerâmica vermelha.

Este tamanho fino de partículas prejudica o processo de sinterização durante a fabricação de aço e é outro motivo para evitar a reciclagem das PSs. A Fig. $2 b$ mostra o diagrama de Winkler [13] com a localização das matérias-primas, bem como algumas composições investigadas neste trabalho. Este diagrama é uma importante ferramenta usada para prever o estágio de extrusão da cerâmica vermelha com base na distribuição de tamanho de partícula dos materiais precursores, onde as regiões numeradas estão associadas a diferentes produtos. De acordo com a Fig. 2b, o bloco de argila está localizado na região 3, apropriado para a fabricação de telhas. A PS está localizada na parte inferior do diagrama perto do canto associado aos tamanhos de partículas superiores a $20 \mu \mathrm{m}$ numa região sem interesse prático para produção cerâmica. A incorporação de PS tende a deslocar o bloco argiloso, BC, para outras regiões, diminuindo sua plasticidade. Isto é devido à diminuição da quantidade de partículas com tamanho inferior a $2 \mu \mathrm{m}$ que estão associadas aos minerais de argila responsáveis pela plasticidade do bloco argiloso [14].

Pode-se observar na Fig. 2b que a quantidade máxima investigada de PS, $20 \%$ em peso, não alterou a localização relativa do bloco de argila, que ainda permanece na região 3 . Esse resultado indica que a PS investigada pode ser incorporada em quantidades maiores que não irão alterar significativamente a trabalhabilidade do BC investigado antes da sinterização para obtenção da cerâmica final.

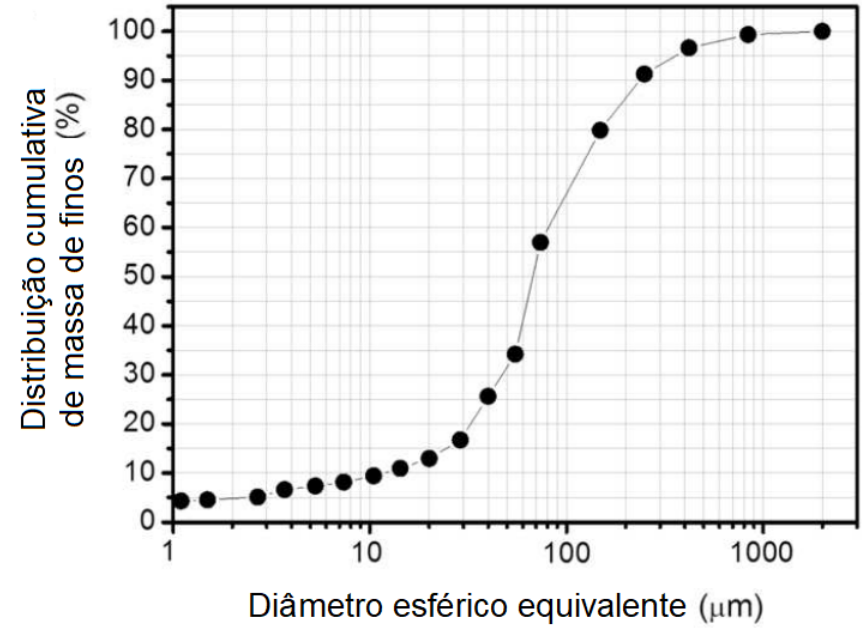

(a)

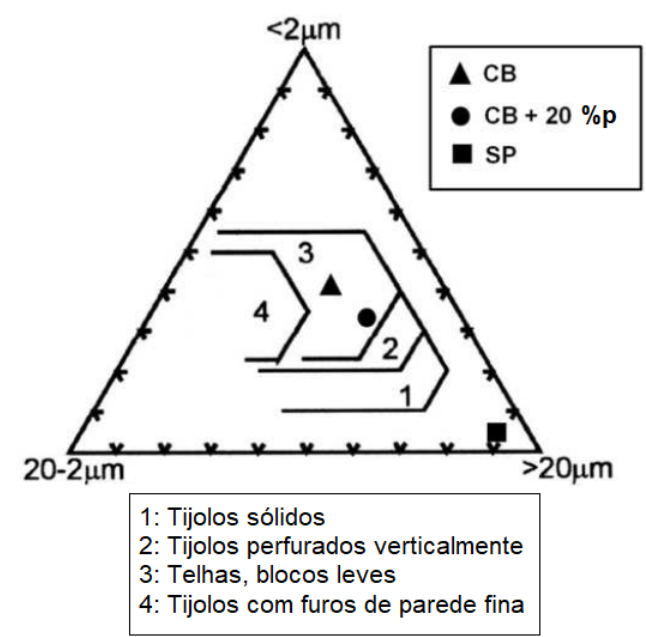

(b)

Figura 2. (a) Distribuição de tamanho de partícula PS (a); e (b) diagrama de Winkler mostrando regiões de aplicação de cerâmica na construção civil. 
A Figura 3 mostra os resultados das propriedades tecnológicas após a sinterização. Esses resultados indicam que a temperatura causa mudanças físicas e mecânicas importantes nas composições investigadas. Houve um aumento em ambas as porosidade (Fig. 3a) e resistência à ruptura flexão (Fig. 3b), principalmente, a $1050^{\circ} \mathrm{C}$. Esses resultados estão associados aos processos de consolidação de cerâmica, bem como às transformações físicas e químicas dos materiais precursores durante os estágios de sinterização [14]. A composição mineralógica da PS sugere um comportamento inerte durante a fase de queima nas temperaturas investigadas. A incorporação da PS tende a aumentar a porosidade do BC em todas as temperaturas investigadas, principalmente a $1050^{\circ} \mathrm{C}$ (Fig. 3a). Este aumento na porosidade contribui para aumentar a absorção de água na cerâmica, que é uma propriedade importante, principalmente para telhas.

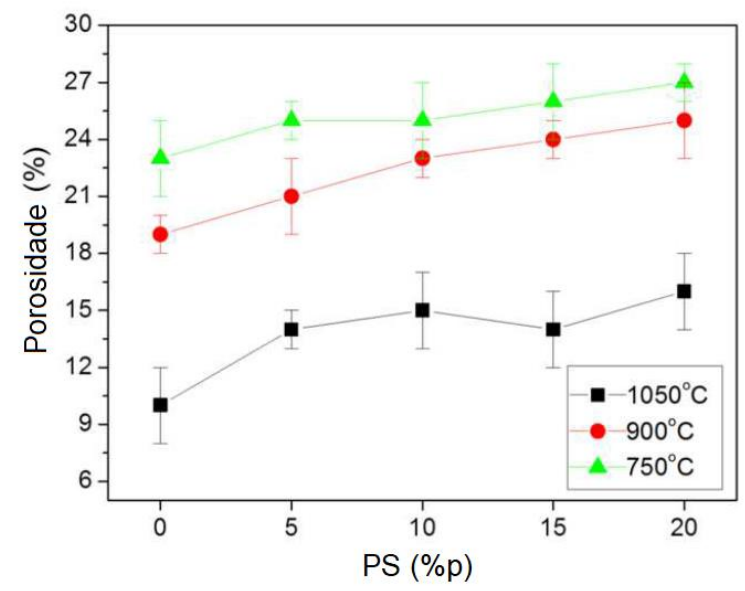

(a)

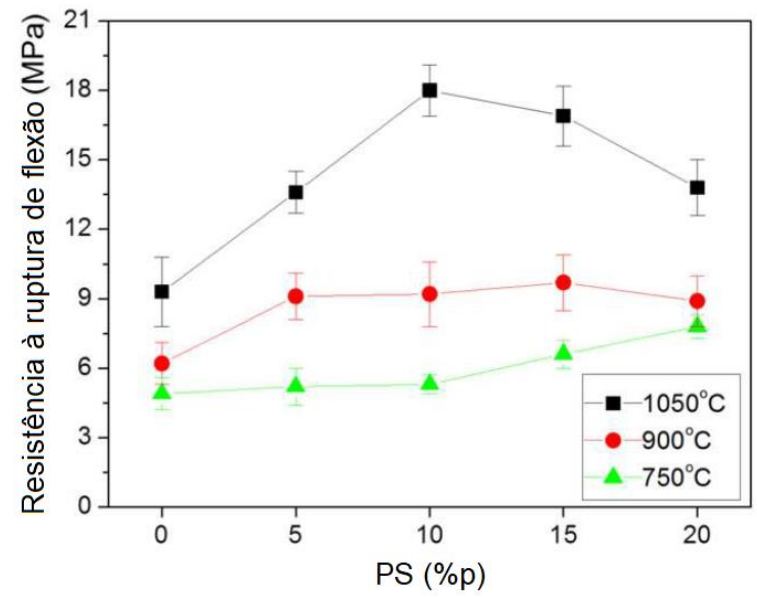

(b)

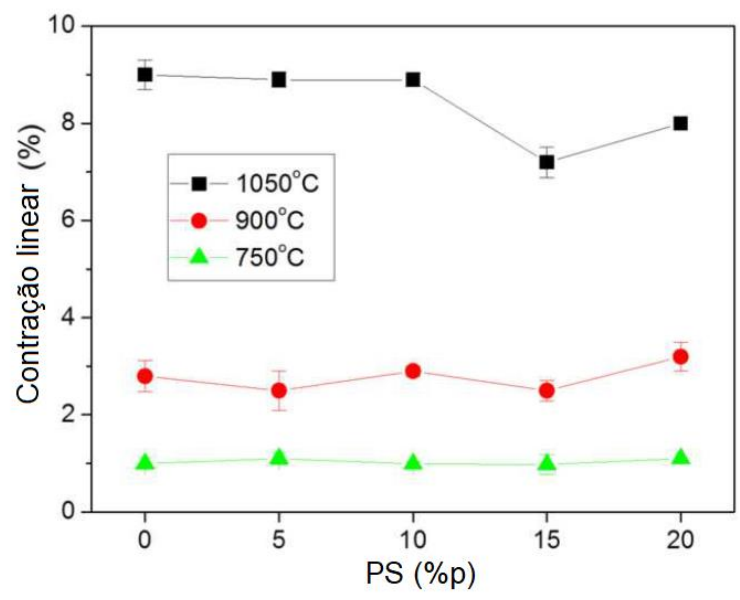

(c)

Figura 3. (a) Porosidade; (b) resistência à ruptura de flexão; e (c) contração linear cerâmica vermelha em função da quantidade de PS incorporada.

A Figura 3b mostra que a resistência à ruptura de flexão da cerâmica vermelha é aumentada com a incorporação de qualquer quantidade de PS a 900 e $1050^{\circ} \mathrm{C}$. Sendo que a $1050^{\circ} \mathrm{C}$, a cerâmica vermelha apresenta um aumento significativo na resistência mecânica até $10 \%$ em peso de incorporação, seguido por uma diminuição moderada. Referente à contração linear (Fig. 3c), observou-se que a incorporação da PS praticamente não altera o valor obtido pela cerâmica vermelha 
em todas as temperaturas. Uma exceção é a da composição com $20 \%$ em peso de PS a $1050^{\circ} \mathrm{C}$, em que ocorre uma diminuição positiva na contração linear.

A composição mineralógica da PS, associada à alta quantidade de compostos de ferro, sugere um comportamento inerte durante a sinterização da cerâmica. Então, a questão é: como poderia, após a queima, mudar a porosidade da cerâmica vermelha com a incorporação da PS? A resposta a esta pergunta provavelmente está relacionada ao empacotamento das amostras durante o estágio de sua formação. Sugere-se que o tamanho de partícula mais grosseiro da PS em comparação com o do bloco de argila, não só aumenta a porosidade, mas também afeta a área de contato das partículas. Uma maior área de contato facilita os mecanismos de sinterização, tanto pela difusão em estado sólido quanto pela formação de fase líquida, o que melhora a resistência da cerâmica [14].

A Figura 4 ilustra uma micrografia de MEV típica da cerâmica vermelha incorporada com $10 \%$ em peso de PS e sinterizada a $1050^{\circ} \mathrm{C}$. Esta foi a condição de maior resistência à flexão (18 $\mathrm{MPa}$ ) e porosidade em torno de $16 \%$. Este resultado mostra, pela primeira vez, evidências do benefício da porosidade nas propriedades mecânicas das cerâmicas vermelhas incorporadas.

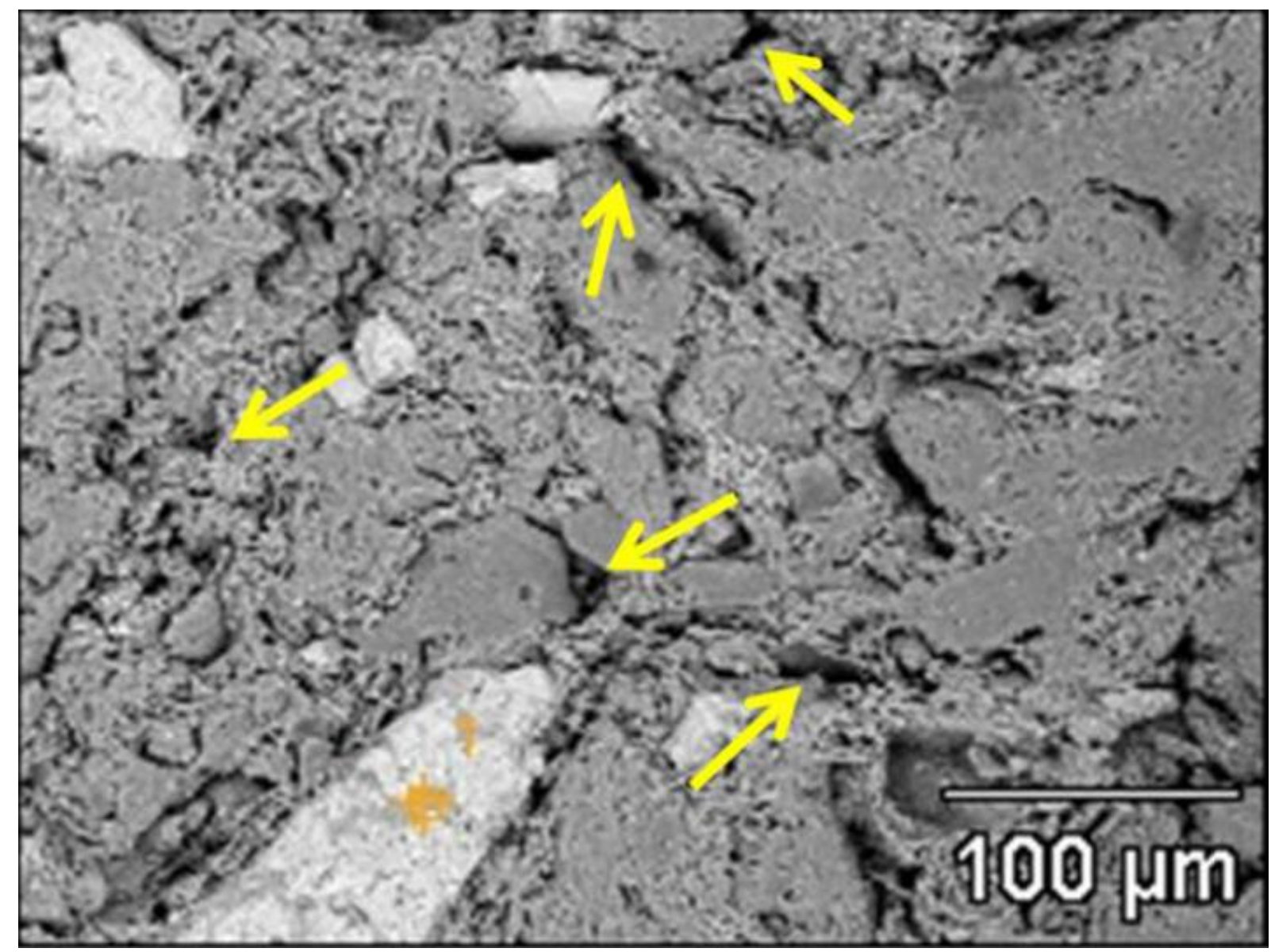

Figura 4. Micrografia de MEV da cerâmica vermelha com a incorporação de $20 \%$ em peso de PS sinterizada a $750^{\circ} \mathrm{C}$ (Setas indicando os poros).

Finalmente, os testes acústicos preliminares e a condutividade térmica resultaram em torno de $0,9 \mathrm{~W} / \mathrm{m} . \mathrm{K}$, indicando que a cerâmica vermelha incorporada com PS apresentou melhores isolamentos térmico e acústico, que são características relevantes para materiais utilizados na construção civil. 


\section{CONCLUSÃO}

- As partículas coletadas no estágio de sinterização de uma aciaria possuem uma quantidade elevada de compostos de $\mathrm{Fe}$ e $\mathrm{Ca}$. As principais fases cristalinas observadas foram hematita, magnetita e calcita. O tamanho de partícula é adequado para sua incorporação direta na fabricação de cerâmica vermelha, produzindo microestrutura porosa;

- A incorporação destas partículas de sínter (PS), em quantidades de até $20 \%$ em peso, em um bloco argiloso não alterou sua capacidade de trabalho e melhorou as propriedades físicas e mecânicas avaliadas das cerâmicas vermelhas sinterizadas. Além disso, a microestrutura porosa da cerâmica contribuiu para melhorar 0 isolamento térmico e acústico, que são características valiosas para a construção civil;

- Os resultados indicaram que as PSs têm potencial para serem utilizadas em cerâmicas vermelhas, pois o aumento da porosidade traz benefícios reais tanto no processamento como na qualidade dos produtos.

\section{Agradecimentos}

Os autores deste trabalho agradecem ao CNPq, à FAPERJ e à CAPES pelo financiamento da pesquisa.

\section{REFERÊNCIAS}

1 Wakelin DH, Fruehan RJ. Making, Shaping and Treating of Steel - Iron Making, 11th ed. Warrendale, PA: AIST Steel Foundation, 1999.

2 Sun W, Zhao L, Li X, Zhao Y. In: Ironmaking and Steelmaking Process: Greenhouse Emissions, Control and Reduction, ed. P. Cavaliere. Switzerland: Springer, 2016.

3 Vieira CMF, Ribeiro MM, Monteiro SN. Microstructural Evaluation of Clayey Ceramic Incorporated with Powder Waste from the Sintering Plant of a Steel-Making Industry. Mater. Sci. Forum. 2012; 727-728:951-956.

4 Ciccu R, Ghiani M, Serci A, Fadda S, Peretti R, Zucca A. Heavy metal immobilization in the mining-contaminated soils using various industrial wastes. Minerals Eng. 2012; 16:187-192.

5 Tsakirids PE, Agatzini-Leonardou S, Oustadakis P. J. Hazar. Mater. 2004; 116:103.

6 Zhang I. Production of bricks from waste materials - a review. Constr. Build. Mater. 2013; 47:643.

7 Monteiro SN, Vieira CMF. On the production of fired clay bricks from waste materials: A critical update. Constr. Build. Mater.2014; 68:599-610.

8 Velasco PM, Ortiz MPM, Giro MAM, Velasco LM. Fired clay bricks manufactured by adding wastes as sustainable construction material - A review. Constr. Build. Mater. 2014; 63:97-107.

9 Quijorna N, Pedro M, Romero M, Andrés A. Characterisation of the sintering behaviour of Waelz slag from electric arc furnace (EAF) dust recycling for use in the clay ceramics industry. J. Environ. Management. 2014; 132:278-286.

10 Monteiro SN, Vieira CMF. Characterization of clays from Campos dos Goytacazes, North Rio de Janeiro State (Brazil). Tile \& Brick Intl. 2002; 18:152-157.

11 Associação Brasileira de Normas Técnicas - ABNT. NBR 7181/84, Determinação da análise granulométrica dos solos. Rio de Janeiro: ABNT, 1984. 
12 American Society for Testing and Materials. ASTM C373-72, Water Absorption, Bulk Density, Apparent Porosity and Apparent Specific Gravity of Fired Whiteware Products. West Conshohocken, PA: ASTM, 1972.

13 Winkler HGF. Keramischen Gesellschaft. 1954; 31: 337.

14 Monteiro SN, Vieira CMF. Solid state sintering of red ceramics at lower temperatures. Ceram. Intl. 2004; 30:381-387. 